\title{
Position control of a SMA microgripper
}

\author{
E. Just, M. Kohl and S. Miyazaki ${ }^{1}$ \\ Forschungszentrum Karlsruhe GmbH, IMT, Postfach 3640, 76021 Karlsruhe, Germany \\ ${ }^{1}$ Institute of Materials Science, University of Tsukuba, Tsukuba, ibaraki 305-8573, Japan
}

\begin{abstract}
A SMA microgripper system with an integrated optical position sensor is presented. The microgripper consists of two actuation units and an optical slit fabricated from one piece of SMA material with overall dimensions of $2 \times 5.8 \times 0.23 \mathrm{~mm}^{3}$. The gripper is sandwiched by two substrates with an aligned photodiode and a photosensor, respectively. This transmission setup allows position sensing with a spatial resolution of $1 \mu \mathrm{m}$ per $2.5 \%$ optical signal change. By feedback of the position signal to a PI controller a position error of $0.3 \%$ is achieved. The typical time constant for reaching stationary positions is about $140 \mathrm{~ms}$.
\end{abstract}

\section{INTRODUCTION}

The continuous miniaturization of devices requires appropriate tools for manipulation of microparts. For the realization of active manipulators and grippers, shape memory alloys (SMAs) appear to be particularly suitable, since they allow simple and compact designs, frictionless actuation and high work outputs [1].

First miniaturized SMA grippers consisted of SMA wires [2-5] or plates [6] and were fabricated by conventional assembly technologies. The development of microgrippers requires, however, the use of micromachining technology, similar to microelectronic devices. The base materials for micromachining are SMA thin films or sheets, which have been developed within the last ten years e.g. by magnetron sputtering, cold-rolling and other technologies [7]. By micromachining, many functional units can be realized in a single piece of SMA material by one fabrication step [8]. Recently, this concept has been implemented in the design of a SMA microgripper with antagonistic actuation $[9,10]$. The present paper introduces an extended microgripper system, which includes actuation and position sensing functions.

\section{DESIGN OF THE GRIPPER SYSTEM}

Fig. 1 shows a scheme of the SMA microgripper in open and closed condition. The gripper mainly consists of two actuation units with bond pads for mechanical and electrical connection, a link between both units with an optical slit and two gripping jaws. Actuation unit I comprises a foldedbeam structure and unit II two circular beams. The actuation units are designed to perform motion within the plane. In particular, unit I is an actuator for linear displacements [11]. The required strain is induced by mounting the gripper on a substrate in prestrained condition. The deformation in the beam structures can be controlled by selective electrical heating of the actuation units, which is enabled by a sufficient thermal isolation between the units. By selective heating of actuation unit I above the phase transformation temperature, the folded beams recover their memory shape, which leads to a linear motion of the optical slit towards actuation unit I. Consequently, the circular beams are deformed and the gripping jaws are closed. By selective heating of actuation unit II, the optical slit and gripping jaws move in opposite direction. Thus, actuation units I and II form an antagonistic pair allowing active control of motion in opposite directions.

In order to avoid local stress concentrations in the actuation units upon loading, the lateral widths of the actuation units have been optimized by iterative finite element simulations using the CAO (computer aided optimization) method [12]. Details on the design optimization of the microgripper can be found in [9]. Optimized gripper designs display nearly homogeneous stress profiles along the lateral beam surfaces of the actuation units. In this way, fatigue effects due to 
stress peaks are avoided and a maximum fraction of material contributes to the shape memory effect.

The microgripper system consists of the micromachined SMA gripper including an optical slit, an infrared light emission diode (IR-LED) and a photosensor, which are aligned with respect to each other as shown in Fig. 2. Depending on the actual position of the optical slit, the optical transmission line between the IR-LED and the photosensor is blocked to a certain degree. Therefore, the resulting transmission intensity reflects the actual position of the slit and thus the actual separation between the gripping jaws $S_{2}$.

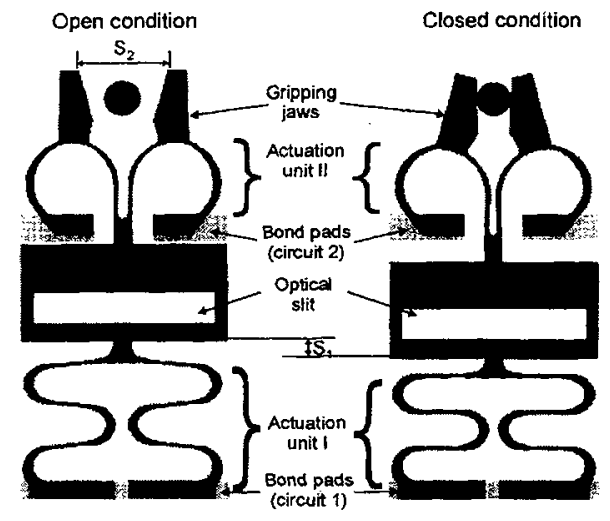

Fig. 1: Operation principle of the SMA microgripper

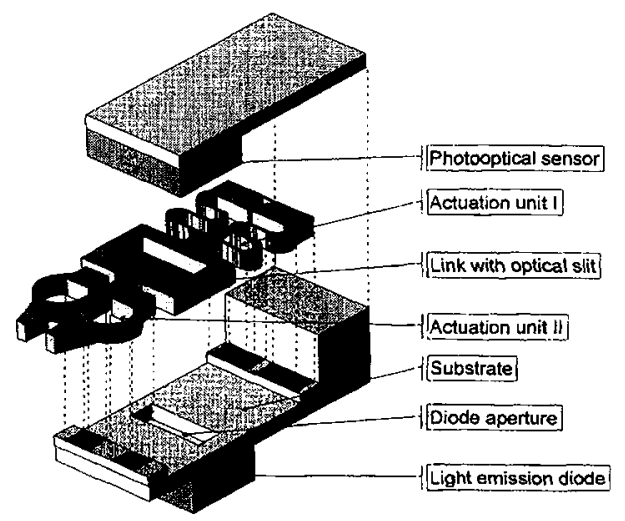

Fig. 2: Scheme of the SMA microgripper system.

\section{MATERIAL PROPERTIES}

The base material for realization of the microgripper has been cold-rolled sheets of an equiatomic TiNi alloy of $230 \mu \mathrm{m}$ in thickness. The one-way shape memory effect has been adjusted by heat treatment in vacuum at $530^{\circ} \mathrm{C}$ for 10 minutes. Phase transformation temperatures were determined by differential scanning calorimetry and electrical resistance measurements. Double-beam test devices were fabricated by laser cutting to characterize the mechanical material properties by beambending experiments. The test devices were loaded by a microweight at the beam end and the corresponding vertical displacements of the beam ends were determined optically by a video microscope. The experiments were performed in a thermostat, which allowed adjustment of the temperature by ambient heating or cooling. Stationary equilibrium conditions have been maintained by cycling the temperature step-wise with sufficient waiting time in every data point. Simultaneously, the electrical resistance of the double-beam devices has been determined by the four-point method.

Fig. 3 shows beam bending and electrical resistance characteristics of a double-beam test device in the transformation temperature regime. Upon cooling, a rhombohedral (R-) phase transformation and a martensitic phase transformation are observed. Upon heating, the martensite directly transforms to the austenitic parent phase. The temperatures of the austenitic, martensitic and $\mathrm{R}$ phase transformation have been determined by the tangential method to $\mathrm{A}_{\mathrm{s}}=56^{\circ} \mathrm{C}, \mathrm{A}_{\mathrm{f}}=78{ }^{\circ} \mathrm{C}$, $M_{s}=26^{\circ} \mathrm{C}, M_{f}=10^{\circ} \mathrm{C}, R_{s}=46^{\circ} \mathrm{C}$ and $R_{f}=38^{\circ} \mathrm{C}$. The indices $s$ and $f$ denote start and finish temperatures, respectively. Since the martensitic transformation starts close to room temperature, mainly the intermediate R-phase transformation is induced by electrical operation above room temperature. This material behavior is of special interest, since R-phase transformations display a narrow hysteresis width of about $1 \mathrm{~K}$ and negligible fatigue effects [13]. For increasing stress level, a further increasing contribution to the actuation occurs due to a stress-induced martensitic transformation. 
(a)

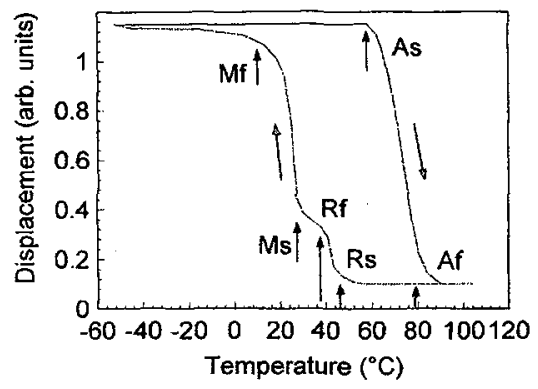

(b)

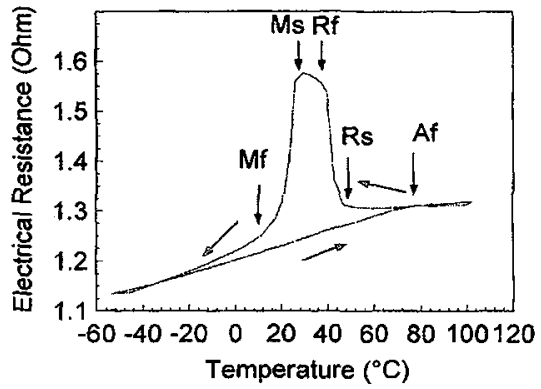

Fig. 3: Beam-bending (a) and electrical resistance characteristic (b) of a double-beam test device. The transformation temperatures are indicated.

\section{FABRICATION}

Since the microgripper system consists of a single piece of SMA material, which combines several functional units, the fabrication procedure is reduced to a few fabrication steps. First, microgrippers are micromachined by laser cutting of a cold-rolled TiNi sheet of $230 \mu \mathrm{m}$ in thickness. Fig. 4 shows a scanning electron micrograph of a microgripper. The lateral size is $2 \times 5.8 \mathrm{~mm}^{2}$. The minimum beam width is $0.05 \mathrm{~mm}$. After the microfabrication step, the microgrippers are prestrained to adjust the maximum stress and strain level for actuation and then mounted on a ceramic substrate by adhesive bonding. Below the optical slit, an opening of $1.8 \times 0.4 \mathrm{~mm}^{2}$ has been cut into the substrate by laser cutting, which serves as an aperture for the IR-LED attached to the substrate from the bottom side. A photooptical sensor with a sensing area of $2 \times 2 \mathrm{~mm}^{2}$ is mounted above the optical slit.

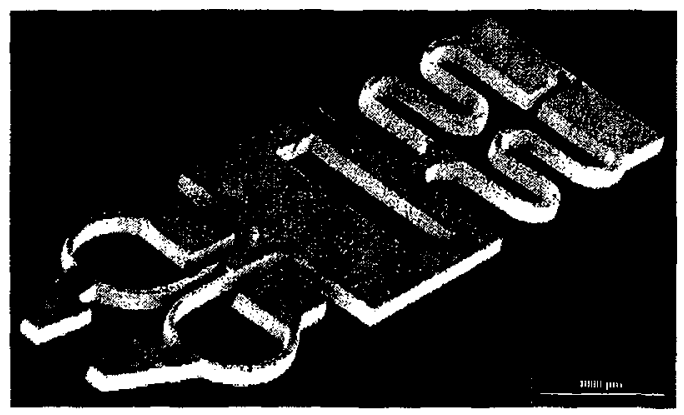

Fig. 4: SMA microgripper

\section{RESULTS AND DISCUSSION}

\subsection{Performance of the actuation units}

Separate actuation units have been realized to investigate their mechanical and electrical properties. A typical force-displacement characteristic of actuation unit II is shown in Fig. 5. The actuation unit has been mounted in vertical orientation in order to attach calibrated weights as illustrated in the inset. Zero displacements $S_{2}$ correspond to completely closed gripping jaws. This condition is reached at zero electrical power for external loads above about $80 \mathrm{mN}$. At smaller loads, the gripping jaws cannot be closed completely. The displacement of the gripper jaws increases for increasing electrical power. For a force of $88 \mathrm{mN}$, the stroke of the gripping jaws $S_{2}$ reaches a maximum value of about $300 \mu \mathrm{m}$. The corresponding vertical displacement of the weight $S_{1}$ is about 
$130 \mu \mathrm{m}$. The maximum gripping force depends on the degree of prestrain before mounting the gripper. By prestraining of actuation unit I by about $130 \mu \mathrm{m}$, the gripping force is estimated to $35 \mathrm{mN}$.

Due to R-phase transformation, the electrical resistance shows a narrow hysteresis for a heating power above $20 \mathrm{~mW}$. At small heating power below $5 \mathrm{~mW}$, the martensitic phase transformation begins to occur. In contrast to the electrical resistance, a pronounced hysteresis of the corresponding displacement characteristics $S_{2}$ is observed, which tends to increase with increasing load. The hysteresis broadening is due to stress-induced martensite (SIM), which is formed locally in the regions of maximum stress along the beam surfaces. These regions mainly determine the observed displacements. Similar hysteresis effects are observed for actuation unit I. From the data of Fig. 5, the corresponding displacement-resistance characteristic shown in Fig. 6 is determined.

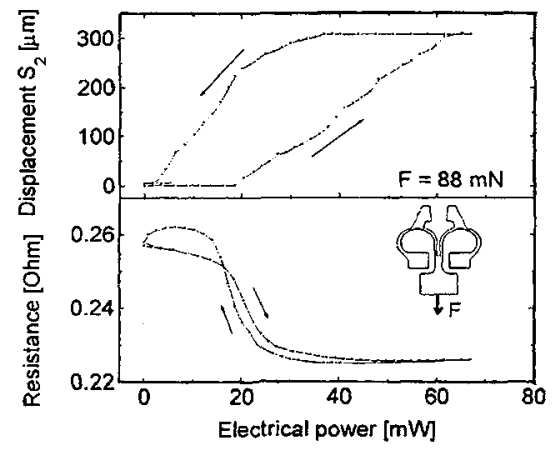

Fig. 5: Electrical and mechanical characteristic of actuation unit II.

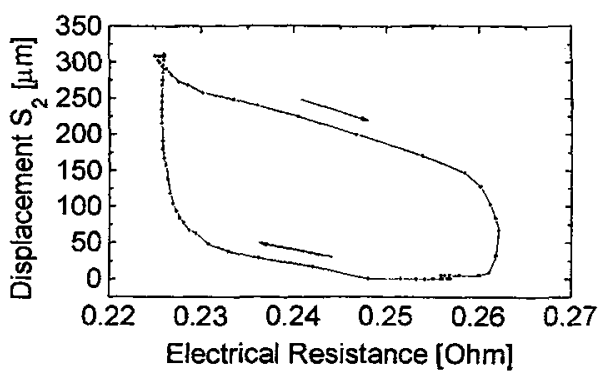

Fig. 6: Displacement-resistance characteristic of actuation unit II.

The rather large hysteresis of the observed gripper displacements raises the problem of a suitable control method for positioning. Recent investigations of certain TiNi wires showed a linear relationship between length change and electrical resistance, which enables smart actuation by using the sensing capability of the wire itself [5]. However, this feature strongly depends on the thermomechanical treatment of the material and on the load-dependent stress distribution in the SMA actuator. In particular, SMA microdevices like the microgripper generally display a nonuniform stress- and temperature distribution during actuation $[9,10]$, which is associated with a nonuniform distribution of martensitic or thombohedral volume fractions.

Nonuniform distributions of martensite or R-phase affect the electrical resistance and mechanical displacement in a different way. In the present case, stress-induced martensite (SIM) occurs in the regions of maximum stress along the beam surfaces, which causes a hysteresis broadening in the displacement characteristic. The corresponding electrical resistance characteristic remains nearly unaffected, since it mainly reflects the average resistance of the whole SMA device rather than local resistance changes. Consequently, the displacement-resistance characteristic generally displays a considerable hysteresis, as shown in Fig. 6, and cannot be used for position control.

\subsection{Performance of the microgripper system}

Another possibility to realize position control is the integration of an additional displacement sensor in the gripper system. Preliminary investigations showed that determination of displacements by a light barrier provides a suitable method, which is rather insensitive to temperature changes in the actuation units and to alignment tolerances of the fabrication process. Fig. 7 shows the optical transmission signal as a function of diplacement of the optical slit, which has been moved by a precision stage in this experiment. Within the range of motion an almost linear characteristic is obtained. In this case, a signal change of $2.5 \%$ corresponds to a spatial resolution of $1 \mu \mathrm{m}$. Since the 
statistical error of the optical measurement is below $2 \%$, displacements can be determined with an accuracy of below $1 \mu \mathrm{m}$, which is sufficient for gripping of objects in the order of $100 \mu \mathrm{m}$ size.

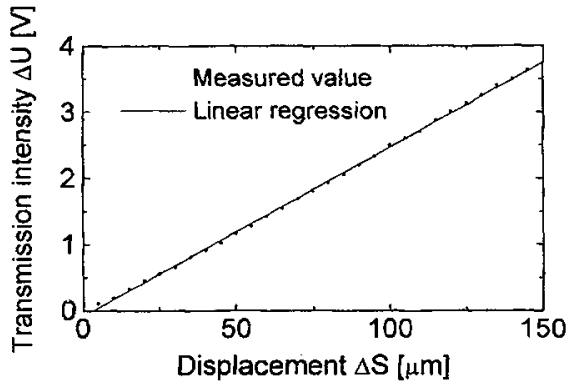

Fig. 7: Calibration characteristic of the optical transmission setup.

Control tests were performed with a PC using a data aquisition board. Analog sensor signals have been processed by a PI control algorithm using LabVIEW. The PI control parameters have been determined by an empirical routine. The maximum power to drive the actuation units has been limited to $80 \mathrm{~mW}$, which corresponds to a maximum control signal of $10 \mathrm{~V}$. The control frequency was $125 \mathrm{~Hz}$. A typical sequence of control signals is shown in Fig. 8 for a stepwise change of the set value. At intermediate position, the control switches between the actuation units. Negative control values correspond to actuation unit I and positive control values to unit II.

In Fig. 8, the initial position is maintained by an average control signal of $2.5 \mathrm{~V}$. After change of the set value, the control system generates a maximum control signal for actuation $I$, which lasts about $120 \mathrm{~ms}$. Then, within the next $20 \mathrm{~ms}$, a stationary average control signal is reached. The corresponding change of the actual displacement takes about $140 \mathrm{~ms}$. The difference between set value and the stationary actual value is about $0.3 \%$. The actual value is kept with an accuracy better than $\pm 0.25 \mu \mathrm{m}$.
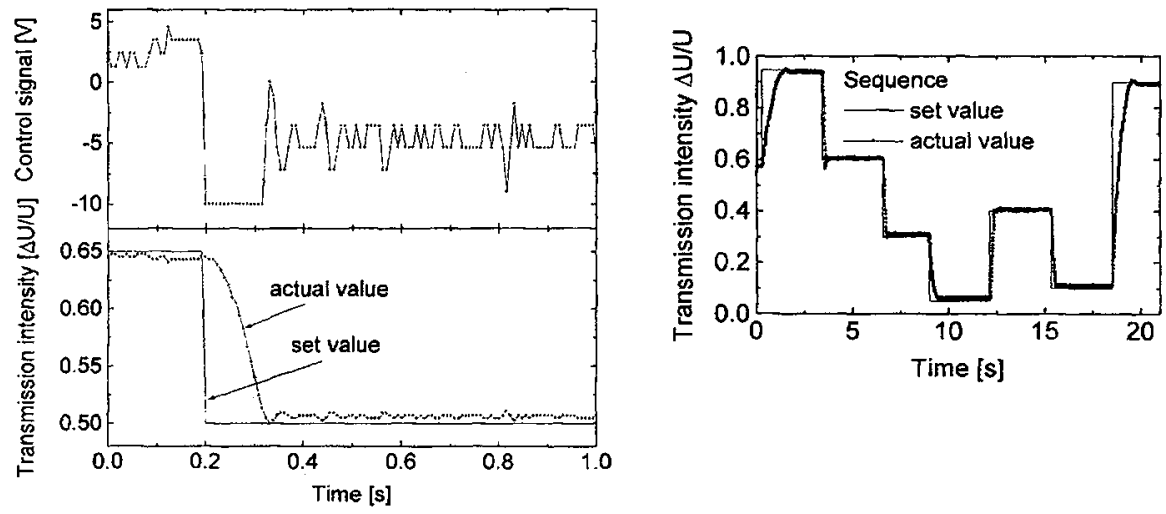

Fig. 8: Control signal and transmission intensity for PI- Fig. 9: Positioning sequence. control.

These features are representative for almost the entire displacement range except for displacements close to the maximum and minimum gripper positions, where longer time constants are observed. Fig. 9 shows the gripper response to an arbitrary sequence of set values for various gripper positions. Close to the end positions small oscillations of the actual position occur and longer time constants are required which are determined by the maximum control power in the experiment. 


\section{CONCLUSIONS}

A microgripper system is presented, which comprises antagonistic actuation and optical sensing functions. The main component of the gripper system is a micromachined SMA device, which consists of several functional units fabricated in one micromachining step from a single sheet of SMA material. This concept provides a cheap and compact solution for microrobotic tasks with high demands on smallness and work output. Possible applications are the handling of microparts of complex shape in cleanroom or vacuum environments, where adhesive or vacuum gripping is not suitable, or in restricted environments with difficult access.

The presented prototype allows a maximum stroke of $300 \mu \mathrm{m}$ and a maximum gripping force of $35 \mathrm{mN}$. The used optical displacement sensor allows a spatial resolution of $1 \mu \mathrm{m}$ per $2.5 \%$ optical signal change. By closed loop positioning with a PI controller a position error of $0.3 \%$ of the full range is archived. The typical time constant for reaching stationary positions is about $140 \mathrm{~ms}$.

\section{Acknowledgements}

The authors would like to thank Gamer Lasertechnik GmbH for fast support.

\section{References}

[1] M. Kohl, Shape memory actuators in MEMS, Proc. SMST 99, Antwerp Zoo, Belgium, (1999).

[2] D. Honma, Y. Miwa and N. Iguchi, Application of shape memory effect to digital control actuator, Bulletin of JSME, Vol. 27, No. 230 (1984), pp. 1737-1742.

[3] K. Kuribayashi, A new actuator of joint mechanism using TiNi alloy wire, Int. J. Robotics Res. 4 (1986), pp. 47-58.

[4] K. Kuribayashi, Millimeter-sized joint actuator using a shape memory alloy, Sensors and Actuators 20 (1989), pp. 57-64.

[5] J. Hesselbach, R. Pittschellis, H. Stork, E. Hornbogen and M. Mertmann, Optimization and control of electrically heated shape memory actuators, Proc. ACTUATOR 94, Bremen, Germany, H. Borgmann and K. Lenz Eds., 1994, pp. 337-340.

[6] K. Kuribayashi, Microrobot using reversible SMA actuator, J. of Robotics and Mechatronics Vol. 3, No. 1 (1990) pp. 52-56.

[7] S. Miyazaki and M. Kohl, Recent developments in TiNi-based shape memory alloys, Int. Symp. on Smart Structures and Materials, San Diego, USA, SPIE Vol. 3324, (1998), p. 2.

[8] M. Kohl, E. Quandt, A. Schüßler, R. Trapp, and D.M. Allen, Characterization of NiTi shape memory microdevices produced by microstructuring of etched sheets or sputter deposited films, Proc. Actuator 94, Bremen, Germany, H. Borgmann and K. Lenz Eds, (1994), pp. 317-320.

[9] E. Just, M. Kohl, W. Pfleging and S. Miyazaki, SMA microgripper with integrated antagonism, Proc. Transducers 99, Sendai, Japan (1999), pp. 1768-1771.

[10] E. Just, M. Kohl, W. Pfleging and S. Miyazaki, SMA microgripper, Proc. SMST 99, Antwerp Zoo, Belgium, (1999).

[11] M. Kohl and K.D. Skrobanek, Linear actuators based on the shape memory effect, Sensors and Actuators, A 70 (1998) 104-111.

[12] C. Mattheck and S. Burkhardt, A new method of structural shape optimization based on biological growth, Int. J. Fatigue, Vol. 12 (1990) 185-190.

[13] S. Miyazaki and K. Otsuka, Deformation and transition behavior associated with the R-phase in TiNi alloys, Metall. Trans. A, Vol. 17A (1986) pp. 53-63. 